\title{
On a Family of Line-Critical Graphs ${ }^{1}$
}

\author{
By \\ Michael D. Plummer, Ann Arbor, Mich., USA \\ With 1 Figure
}

(Received December 20, 1965)

\section{Introduction}

A set of points $M$ of a graph $G$ is a point-cover if each line of $G$ is incident with at least one point of $M$. A minimum cover, abbreviated $\mathrm{m}$. c., for $G$ is a point cover with a minimum number of points. The point covering number of $G, \alpha(G)$, is the number of points in any minimum cover of $G$. If $x$ is a line in $G$, we denote by $G-x$ the graph obtained by deleting $x$. A line $x$ is said to be critical (with respect to point-cover) if $\alpha(G-x)<\alpha(G)$. Ore [4] mentions such critical lines and in [3], we consider the case where the graph involved is a tree. In particular, if each line of the graph $G$ is critical, $G$ is said to be line-critical. It is obvious that all odd cycles are line-critical graphs as are all complete graphs. Erdös and Gallai [2] obtain a bound on the number of lines in such a graph in terms of the point covering number. A structural characterization of this family of graphs is, however, presently unknown. In an earlier paper [1], we show that if two adjacent lines of a graph are both critical, then they must lie on a common odd cycle. Hence, in particular, a line-critical graph is a block in which every pair of adjacent lines lie on a common odd cycle.

In this paper we shall develop an infinite family of line-critical graphs. This family includes all graphs known by the author to be line-critical and in particular it includes all those line-critical graphs with fewer than eight points.

1 Work supported in part by the U. S. Air Force Office of Scientific Research under Grant AF-AFOSR-754-65. 


\section{Additional Terminology}

A graph $G$ consists of a finite set of points $V(G)$ together with a collection of lines $E(G)$ each of which is an unordered pair of points. If $x$ is the line containing points $u$ and $v$, then we write $s=u v$. If two points (lines) are joined by a line (point), we say that the points (lines) are adjacent. If a point and a line meet, we say they are incident. The degree of a point $v, d(v)$, is the number of lines ineident with it. If $d(v)=0$, we say $v$ is an isolated point. A path joining points $u$ and $v$ is an alternating sequence of distinct points and lines beginning with $u$ and ending with $v$ so that each line is incident with the point before it and the point after it.

A cycle is a path containing more than one line together with an additional line joining the first and last points of the path. The length of a path or a cycle is the number of lines in it and a cycle is said to be even $(o d d)$ if its length is even (odd). A graph $G$ is said to be connected if every two distinct points in $G$ are joined by a path. $A$ point $v$ is a cutpoint of a connected graph $G$ if the graph obtained from $G$ by deleting $v$ is disconnected. A subgraph of $G$ is a block of $G$ if $B$ is a maximal connected subgraph of $G$ having no cutpoints. Finally, let $|A|$ denote the number of elements in the set $A$.

\section{The Construction}

Let $v_{0}$ be a point of a connected graph $G$ with $d\left(v_{0}\right)>1$. For any $p \geqslant 3$, let $K_{p}-x$ denote the complete graph on $p$ points with any one line $x$ deleted, where $x=u_{1} u_{2}$. We construct a new graph as follows. Split $G$ at $v_{0}$ forming two new points $v_{1}$ and $v_{2}$, retaining all lines from $G$ and adding no new lines. Furthermore, we make the restriction that neither $v_{1}$ nor $v_{2}$ is an isolated point in the new graph. Beyond this, however, no restriction is made on how the set of lines incident with $v_{0}$ is divided between $v_{1}$ and $v_{2}$. There are of course $2^{d\left(v_{0}\right)}-2$ graphs obtainable from $G$ in this fashion, some of which may be isomorphic. If $d\left(v_{1}\right)=m$ and $d\left(v_{2}\right)=n$, we denote any of the graphs obtained above by $S\left(G, v_{0} ; m, n\right)$. Next, we attach $K_{p}-x$ to $S\left(G, v_{0}\right.$; $m, n$ ) by identifying $u_{1}$ with $v_{1}$ and $u_{2}$ with $v_{2}$. The resulting graph is denoted by $S\left(p ; G, v_{0} ; m, n\right)$. We illustrate this construction in Figure 1. 

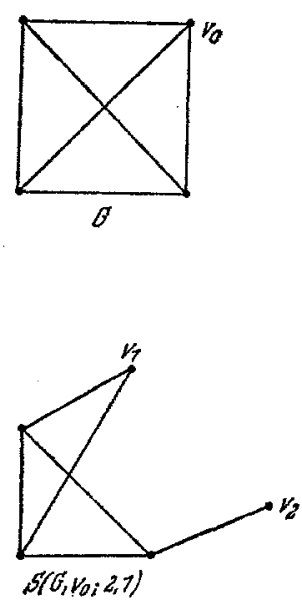
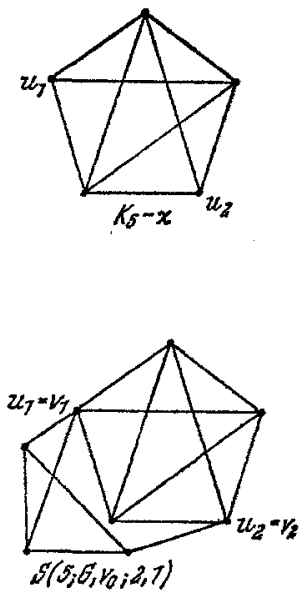

Fig. 1

We now proceed to show that if one performs the above construction, a resulting graph $S\left(p ; G, v_{0} ; m, n\right)$ is line-critical if and only if $G$ is line-critical.

Theorem 1. If $v$ is a point incident with a critical line of a graph $G$, then $G$ has minimum covers $M_{1}, M_{2}$ such that $v \in M_{1}-M_{2}$.

Proof. Let $M_{x}$ be an m.c. for $G-x$ and suppose $x=u v$ is a critical line of $G$. Now since $x$ is critical, neither $u$ nor $v$ is in $M_{x}$. Let $M_{1}=M_{x} \cup\{v\}$ and $M_{2}=M_{x} \cup\{u\}$. Then $M_{1}$ and $M_{2}$ each cover $G$ and since $\left|M_{1}\right|=$ $=\left|M_{x}\right|+1=\alpha(G)=\left|M_{2}\right|$, each is a minimum cover. This completes the proof.

Our next theorem relates the point covering numbers of $G$ and of $S\left(p ; G, v_{0} ; m, n\right)$.

Theorem 2. If $v_{0}$ is a point of graph $G$ and $d\left(v_{0}\right)>1$ and if $p \geqslant 3$, then $\alpha\left[S\left(p ; G, v_{0} ; m, n\right)\right]=\alpha(G)+p-2$.

Proof. Suppose $G$ has an m.c. $M$ which contains $v_{0}$. Let $W$ be any m.c. for $K_{p}$ which includes $u_{1}$ and $u_{2}$. Then $\left[M-\left\{v_{0}\right\}\right] \cup W$ covers $S\left(p ; G, v_{0} ; m, n\right)$ and thus $\alpha\left[S\left(p ; G, v_{0} ; m, n\right)\right] \leqslant\left|M-\left\{v_{0}\right\}\right|+|W|=$ $=|M|-1+(p-1)=\alpha(G)+p-2$.

Now suppose $G$ has an m.c. $\boldsymbol{M}^{\prime}$ which does not contain $v_{0}$. Let $W^{\prime}=V\left(K_{p}\right)-\left\{u_{1}, u_{2}\right\}$. Clearly, $W^{\prime}$ is the m.c. for $K_{p}-x$. Hence $M^{\prime} \cup W^{\prime}$ covers $S\left(p ; G, v_{0} ; m, n\right)$ and we have 


$$
\begin{aligned}
\alpha\left[S\left(p ; G, v_{0} ; m, n\right)\right] \leqslant\left|M^{\prime} \cup W^{\prime}\right| & =\left|M^{\prime}\right|+\left|W^{\prime}\right|= \\
& =\alpha(G)+\alpha\left(K_{p}-x\right)=\alpha(G)+p-2 .
\end{aligned}
$$

Thus in either case, one obtains the inequality:

(1) $\alpha\left[S\left(p ; G, v_{0} ; m, n\right)\right] \leqslant \alpha(G)+p-2$.

Now let $M$ be an m.c. for $S\left(p ; G, v_{0} ; m, n\right)$. We have three cases to consider.

(i) Suppose $\left\{u_{1}, u_{2}\right\} \cap M=\phi$. Then all points of $K_{p}$ other than $u_{1}$ and $u_{2}$ are in $\boldsymbol{M}$. Also, if $u_{1} w_{i}, i=1, \ldots, m$ are the lines of $G$ incident with $u_{1}$ and if $u_{2} w_{i}, i=m+1, \ldots, m+n$, are those lines incident with $u_{2}$, then $\left\{w_{1}, \ldots, w_{m}, w_{m+1}, \ldots, w_{m+n}\right\} \subset M$. Hence $M-\left[V\left(K_{p}\right)-\left\{u_{1}, u_{2}\right\}\right]$ covers $G$. Hence $\alpha(G) \leqslant \mid M-\left[V\left(K_{p}\right)-\right.$ $\left.-\left\{u_{1}, u_{2}\right\}\right]|=| M \mid-(p-2)=\alpha\left[S\left(p ; G, v_{0} ; m, n\right)\right]-p+2, \quad$ and thus $\alpha\left[S\left(p ; G, v_{0} ; m, n\right)\right] \geqslant \alpha(G)+p-2$.

(ii) Next suppose $\left\{u_{1}, u_{2}\right\} \subset M$. Then clearly $M \cap\left[V(G)-\left\{v_{0}\right\}\right]$ is an m.c. for $G-v_{0}$. Also in this case, $\left|M \cap V\left(K_{p}-x\right)\right|=p-1$. Thus $\alpha\left[S\left(p ; G, v_{0} ; m, n\right)\right]=|M|=\left|M \cap V\left(K_{p}-x\right)\right|+\left|M \cap V\left(G-v_{0}\right)\right|=$ $=p-1+\alpha\left(G-v_{0}\right) \geqslant p-2+\alpha(G)$ and thus

$$
\alpha\left[S\left(p ; G, v_{0} ; m, n\right)\right] \geqslant \alpha(G)+p-2 .
$$

(iii) Finally, suppose $u_{1} \in M, u_{2} \notin M$. Now every point of $K_{p}-x$, except $u_{2}$, is in $M$, since all such points are adjacent to $u_{2}$. Let $w$ be a point of $K_{p}-x, w \neq u_{1}$. Then $w \in M$ and $M_{0}=[M-\{w\}] \cup\left\{u_{2}\right\}$ covers $S\left(p ; G, v_{0} ; m, n\right)$. Since $\left|M_{0}\right|=|M|, M_{0}$ is a minimum cover for $S\left(p ; G, v_{0} ; m, n\right)$ and $u_{1}, u_{2} \in M_{0}$. Hence by (ii), we again obtain $\alpha\left[S\left(p ; G, v_{0} ; m, n\right)\right] \geqslant \alpha(G)+p-2$. Thus all three cases give rise to the inequality:

(2) $\alpha\left[S\left(p ; G, v_{0} ; m, n\right)\right] \geqslant \alpha(G)+p-2$.

This, together with inequality (1) yields $\alpha\left[S\left(p ; G, v_{0} ; m, n\right)\right]=$ $\alpha(G)+p-2$ and the theorem is proved.

We are now prepared to prove the main theorem concerning this construction.

Theorem 3. If $v_{0}$ is a point of degree at least 2 of a graph $G$ and if $p \geqslant 3$, then $G$ is line-critical if and only if $S\left(p ; G, v_{0} ; m, n\right)$ is line-critical.

Proof. Suppose that $G$ is a line-critical graph. Let $x=u_{1} u_{2}$ be the line deleted from $K_{p}$. Let $y$ be any line of $S\left(p ; G, v_{0} ; m, n\right)$. We shall consider four cases. 
(i) Suppose $y$ is a line of $G$ incident with $v_{0}$. Let $M_{y}$ be an m.c. for $G-y$. In this proof we shall denote the set of lines in $G$ incident with $v_{0}$ by $v_{0} w_{i}, i=1, \ldots, r$. Hence we may assume without loss of generality in this case that $y=v_{0} w_{1}$. Then $v_{0}, w_{1} \notin M_{y}$ and hence $w_{2}, \ldots, w_{r} \in M_{y}$. Then $M_{y} \cup\left[V\left(K_{p}\right)-\left\{u_{1}, u_{2}\right\}\right]$ covers $S\left(p ; G, v_{0} ; m, n\right)-y$. Thus

$$
\begin{gathered}
\alpha\left[S\left(p ; G, v_{0} ; m, n\right)-y\right] \leqslant\left|M_{y} \cup\left[V\left(K_{p}\right)-\left\{u_{1}, u_{2}\right\}\right]\right|= \\
=\left|M_{y}\right|+\left|V\left(K_{p}\right)-\left\{u_{1}, u_{2}\right\}\right|=\alpha(G-y)+p-2= \\
=\alpha(G)+p-3=\alpha\left[S\left(p ; G, v_{0} ; m, n\right)\right]-1
\end{gathered}
$$

by Theorem 2 . Hence $y$ is critical in $S\left(p ; G, v_{0} ; m, n\right)$.

(ii) Suppose $y$ is a line of $G$ which is not incident with $v_{0}$. Again let $M_{y}$ be an m.c. for $G-y$. If $v_{0} \notin M_{y}$, then $\left\{w_{2}, \ldots, w_{r}\right\} \subset M_{y}$ and $M_{y} \cup\left[V\left(K_{p}\right)-\left\{u_{1}, u_{2}\right\}\right]$ again covers $S\left(p ; G, v_{0} ; m, n\right)-y$ and as in case (i) $y$ is critical in $S\left(p ; G, v_{0} ; m, n\right)$. If $v_{0} \in M_{y}$, then $\left[M_{y}-\left\{v_{0}\right\}\right] \cup W$ covers $S\left(p ; G, v_{0} ; m, n\right)-y$, where $W$ is any m.c. for $K_{p}$ which contains $u_{1}$ and $u_{2}$. Thus $\alpha\left[S\left(p ; G, v_{0} ; m, n\right)-y\right] \leqslant\left|M_{y}-\left\{v_{0}\right\}\right|+|W|=$ $=\left|M_{y}\right|-1+p-1=\alpha(G-y)+p-2=\alpha(G)+p-3=$ $=\alpha\left[S\left(p ; G, v_{0} ; m, n\right)\right]-1$ by Theorem 2 and hence again $y$ is critical in $S\left(p ; G, v_{0} ; m, n\right)$.

(iii) In this case we assume that $y$ is a line in $K_{p}-x$ incident with $u_{1}$ or $u_{2}$. Without loss of generality, let $y=u_{1} w_{0}$. Let $z$ be any line of $G, z=v_{0} w_{k}$, incident with $v_{0}$ in $G$ and with $u_{2}$ in $S\left(p ; G, v_{0} ; m, n\right)$. Now $z$ is critical in $G$, hence there is an m.c. $M_{z}$ for $G-z$, such that $v_{0}, w_{k} \notin M_{z}$, and $w_{j} \in M_{z}$ for $j \neq k$. Let $M_{0}=M_{z} \cup\left[V\left(K_{p}\right)-\left\{u_{1}, w_{0}\right\}\right]$. Then $M_{0}$ covers $S\left(p ; G, v_{0} ; m, n\right)-y$. Hence

$\alpha\left[S\left(p ; G, v_{0} ; m, n\right)-y\right] \leqslant\left|M_{0}\right|=\left|M_{z}\right|+\left|V\left(K_{p}\right)-\left\{u_{1}, w_{0}\right\}\right|=$ $\alpha(G-z)+p-2=\alpha(G)+p-3=\alpha\left[S\left(p ; G, v_{0} ; m, n\right)\right]-1$ and $y$ is critical in $S\left(p, G, v_{0} ; m, n\right)$ again using Theorem 2 .

(iv) Finally let us suppose that $y=v_{3} v_{4}$ is a line in $K_{p}-x$ incident with neither $u_{1}$ nor $u_{2}$. By Theorem 1 , there is an m.c. $M$ for $G$ which contains $v_{0}$. Let $W^{\prime}=V\left(K_{p}\right)-\left\{v_{3}, v_{4}\right\}$. Then $W^{\prime}$ contains $u_{1}$ and $u_{2}$ and hence it covers $\left(K_{p}-x\right)-y$. Hence $\left[M-\left\{v_{0}\right\}\right] \cup W^{\prime}$ covers $S\left(p ; G, v_{0} ; m, n\right)-y$. Thus $\alpha\left[S\left(p ; G, v_{0} ; m, n\right)-y\right] \leqslant\left|M-\left\{v_{0}\right\}\right|+$ $+\left|W^{\prime}\right|=|M|-1+(p-2)=\alpha(G)+p-3=\alpha\left[S\left(p ; G, v_{0} ; m, n\right)\right]-1$ by Theorem 2 and hence $y$ is critical in $S\left(p ; G, v_{0} ; m, n\right)$.

Now assume that $S\left(p ; G, v_{0} ; m, n\right)$ is line-critical. 
First suppose that $y$ is a line in $G$ incident with $v_{0}$. Thus $y$ is incident with $u_{1}$ or $u_{2}$ in $S\left(p ; G, v_{0} ; m, n\right)$, say $u_{1}$. Now $y$ is critical in $S\left(p ; G, v_{0} ; m, n\right)$ and hence there is an m.c. $M^{\prime}$ for $S\left(p ; G, v_{0} ; m, n\right)-y$ with $u_{1} \notin M^{\prime}$ and with $\left[V\left(K_{p}\right)-\left\{u_{1}, u_{2}\right\}\right] \subset M^{\prime}$. Now $M^{\prime}-\left[V\left(K_{p}\right)-\left\{u_{1}, u_{2}\right\}\right]$ covers $G-y$. Hence,

$$
\begin{aligned}
& \alpha(G-y) \leqslant\left|M^{\prime}-\left[V\left(K_{p}\right)-\left\{u_{1}, u_{2}\right\}\right]\right|=\left|M^{\prime}\right|-(p-2) \\
& =\alpha\left[S\left(p ; G, v_{0} ; m, n\right)-y\right]-p+2=\alpha\left[S\left(p ; G, v_{0} ; m, n\right)\right]-p+1 \\
& \leqslant \alpha(G)+p-2-p+1=\alpha(G)-1 \text { by Theorem } 2 \text { and thus } y
\end{aligned}
$$
is critical in $G$.

Finally, suppose $y$ is a line in $G$ which is not incident with $v_{0}$. Let $M^{\prime}$ be an m.c. for $S\left(p ; G, v_{0} ; m, n\right)-y$. If $\left[V\left(K_{p}\right)-\left\{u_{1}, u_{2}\right\}\right] \subset M^{\prime}$, then $y$ is critical in $G$ as before. If $\left[V\left(K_{p}\right)-\left\{u_{1}, u_{2}\right\}\right] \notin M^{\prime}$, then let $w$ be a point in $\left[V\left(K_{p}\right)-\left\{u_{1}, u_{2}\right\}\right]-M^{\prime}$. But $w_{1}$ and $u_{1}$ are adjacent as are $w$ and $u_{2}$. Hence $u_{1}, u_{2} \in M^{\prime}$. Thus $\left|M^{\prime} \cap V\left(K_{p}\right)\right|=p-1$. Now $\left[M^{\prime}-V\left(K_{p}\right)\right] \cup\left\{v_{0}\right\}$ covers $G-y$. Hence

$$
\begin{aligned}
& \alpha(G-y) \leqslant\left|\left[M^{\prime}-V\left(K_{p}\right)\right] \cup\left\{v_{0}\right\}\right|=\left|M^{\prime}-V\left(K_{p}\right)\right|+1 \\
& =\left|M^{\prime}\right|-(p-1)+1=\alpha\left[S\left(p ; G, v_{0} ; m, n\right)-y\right]-p+2 \\
& =\alpha\left[S\left(p ; G, v_{0} ; m, n\right)\right]-p+1 \leqslant \alpha(G)-1 \text { again by Theorem } 2 .
\end{aligned}
$$

Thus $y$ is critical in $G$ and the theorem is proved.

\section{Data on line-eritical graphs}

In Table 1 below, $p$ denotes the number of points and $q$ the number of lines of the corresponding graph.

Table 1. The line-critical graphs with fewer than eight points

\begin{tabular}{c|c|c|cc}
\hline$p$ & 0 & $\alpha(\theta)$ & & $G$ \\
\hline 2 & 1 & 1 & $k_{3}$ & \\
\hline 3 & 3 & 2 & $k_{3}$
\end{tabular}




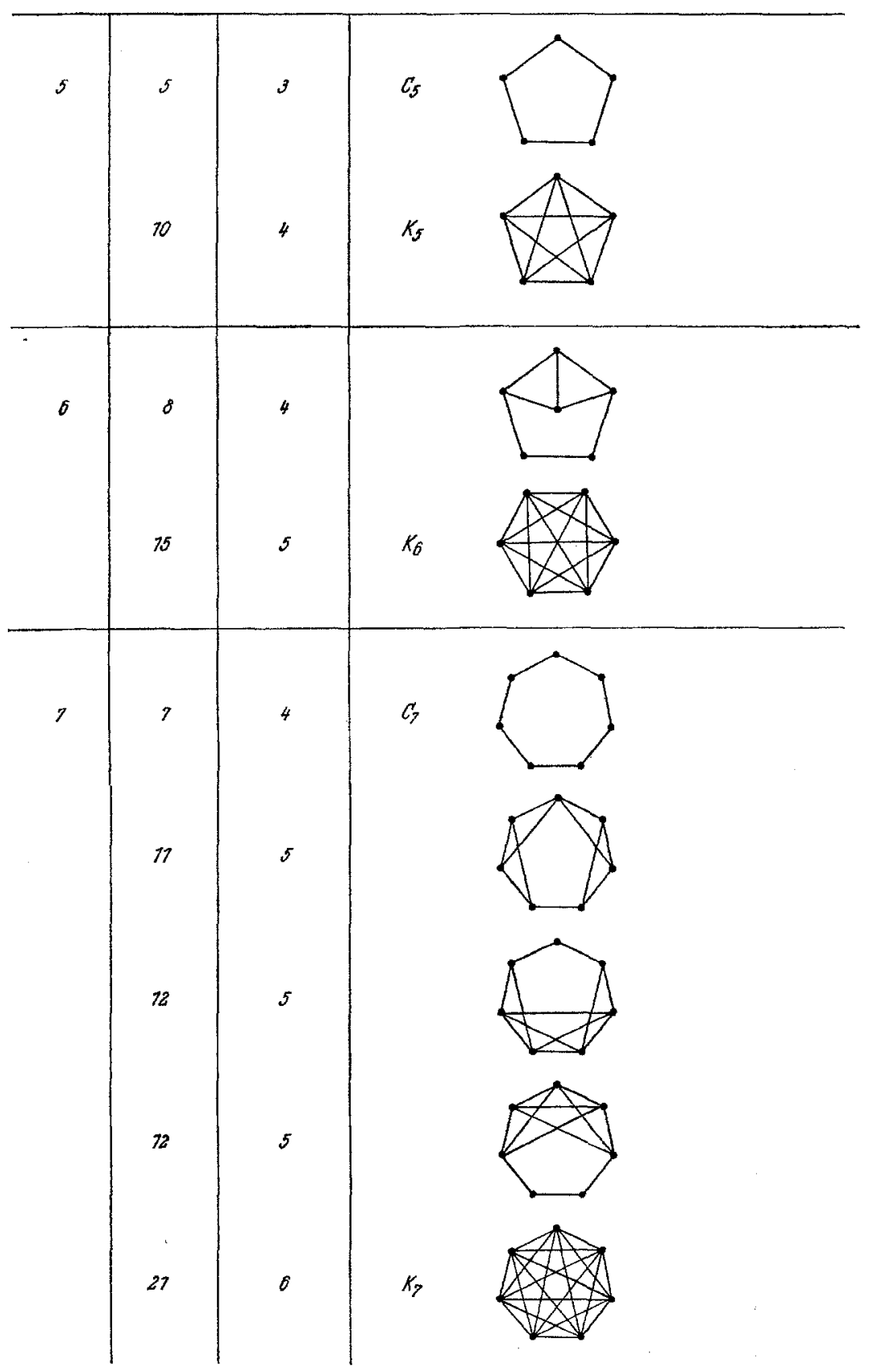


One may obtain each of the graphs in Table 1 by starting with a complete graph and performing a sequence of constructions of the type

Table 2. A family of line-critical graphs with eight points

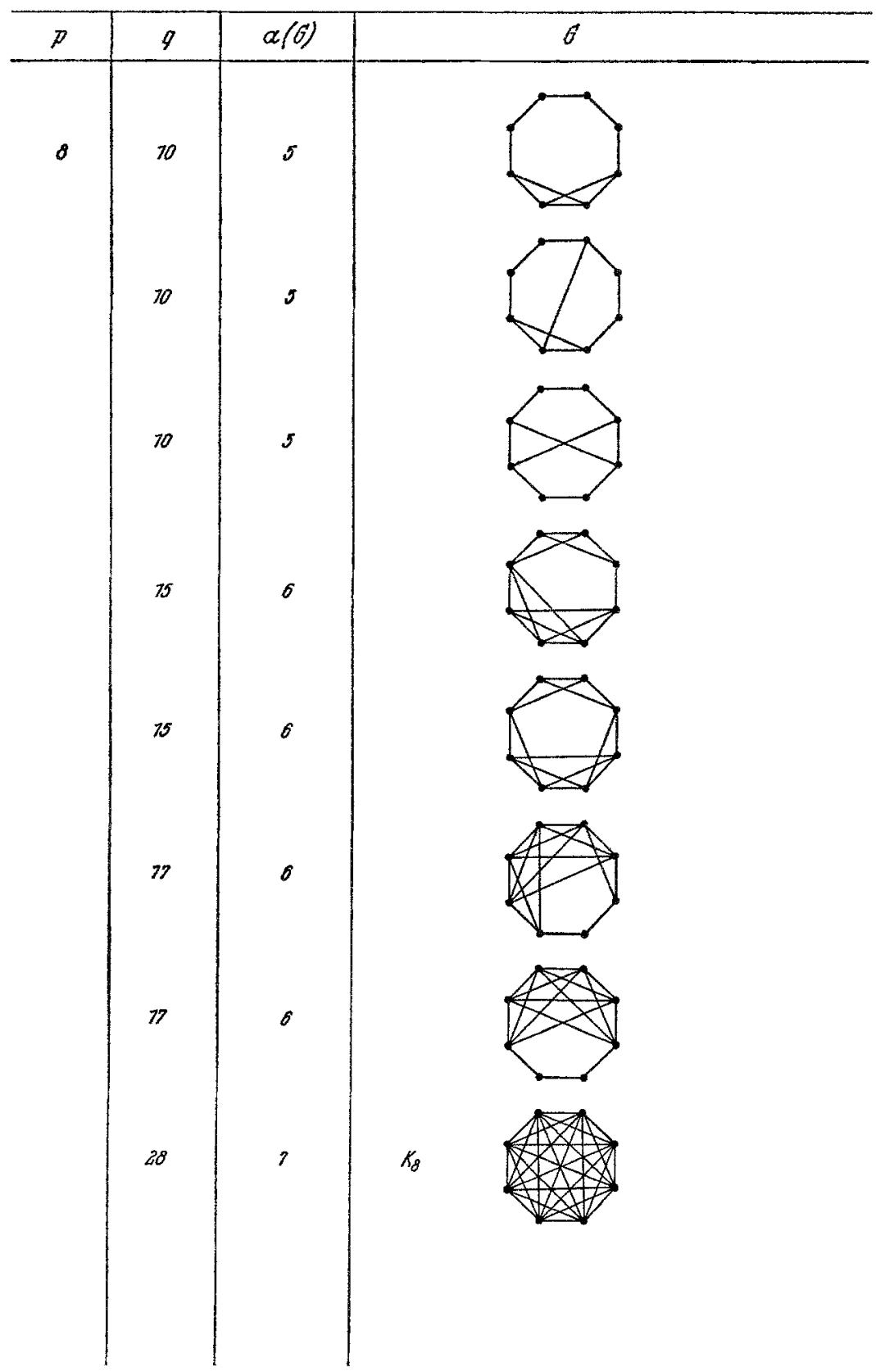


described in Section 3. Hence this construction yields all the linecritical graphs with fewer than eight points. The large number of graphs with eight points has discouraged a direct search for those which are line-critical. In Table 2, however, we present all those line-critical graphs with eight points obtainable by starting with a complete graph and performing a sequence of constructions as in Section 3.

\section{References}

[1] L. W. Beineke, F. Harary, and M. D. Plummer: On the critical lines of a graph, Pacific J. Math., to appear.

[2] P. Erdös and T. Gallai: On the minimal number of vertices representing the edges of a graph, Magyar Tud. Akad. Mat. Kutató Int. Közl., 6 (1961), $89-96$.

[3] F. Harary and M.D. Plummer: On the critical points and lines of a tree, Magyar Tud. Akad. Mat. Kutató Int. Közl., to appear.

[4] O. Ore: Theory of Graphs, Amer. Math. Soc. Colloq. Pub. vol. 38, Providence, 1962 .

The University of Michigan 